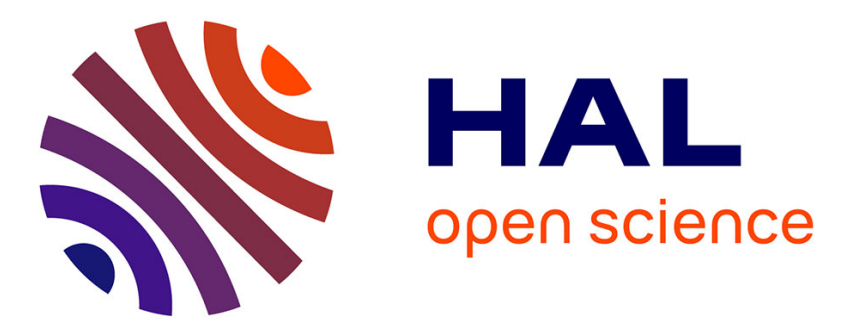

\title{
Brief: Explicit and Tight Bounds of the Convergence Time of Average-based Population Protocols
}

\author{
Yves Mocquard, Bruno Sericola, Emmanuelle Anceaume
}

\section{To cite this version:}

Yves Mocquard, Bruno Sericola, Emmanuelle Anceaume. Brief: Explicit and Tight Bounds of the Convergence Time of Average-based Population Protocols. SIROCCO 2019 - 26th International Colloquium Structural Information and Communication Complexity, Jul 2019, L'Aquila, Italy. pp.1-4, 10.1007/978-3-030-24922-9_29. hal-02380422

\section{HAL Id: hal-02380422 \\ https://cnrs.hal.science/hal-02380422}

Submitted on 26 Nov 2019

HAL is a multi-disciplinary open access archive for the deposit and dissemination of scientific research documents, whether they are published or not. The documents may come from teaching and research institutions in France or abroad, or from public or private research centers.
L'archive ouverte pluridisciplinaire HAL, est destinée au dépôt et à la diffusion de documents scientifiques de niveau recherche, publiés ou non, émanant des établissements d'enseignement et de recherche français ou étrangers, des laboratoires publics ou privés. 


\title{
Explicit and Tight Bounds of the Convergence Time of Average-based Population Protocols
}

\author{
Yves Mocquard* $^{*}$ Bruno Sericola ${ }^{\dagger}$ Emmanuelle Anceaume ${ }^{\ddagger}$
}

\section{Introduction}

This paper focuses on the deep analysis of average-based problems in the population protocol model [1], a model in which agents are identically programmed, with no identity, and they progress in their computation through random pairwise interactions. A considerable amount of work has been done so far to determine which properties can emerge from pairwise interactions between finite-state agents, together with the derivation of bounds on the time and space needed to reach such properties. In this work, we are primarily interested in problems that aim at quantifying properties on the system population, such as the proportion problem [2] or the counting problem [3]. Namely, we consider a set of $n$ agents, interconnected by a complete graph, that asynchronously start their execution in one of two distinct states $A$ (associated with some positive integer $m$ ) and $B$ (associated with 0 ), and such that $n_{A}$ (resp. $n_{B}$ ) is the number of agents whose initial state is $A$ (resp. $B$ ). Such problems can be solved by relying on averagebased population protocols $[4,3,2]$. Briefly, $n$ agents starting independently from each other with an initial integer state, interact randomly by pairs, and at each interaction, keep the average of both states as their new state. Average-based protocols have also been used in gossip-based aggregation protocols as well as in consensus protocols [5].

\section{Average-based Population Protocols}

Average-based population protocols use the average technique to compute the proportion of agents that started their execution in a given state $A$. The notion of time in population protocols refers to as the successive steps at which interactions

${ }^{*}$ Univ. Rennes, CNRS, Inria, IRISA (France), yves.mocquard@irisa.fr

${ }^{\dagger}$ Inria, Univ. Rennes, CNRS, IRISA (France), bruno.sericola@inria.fr

${ }^{\ddagger}$ CNRS, Univ. Rennes, Inria, IRISA (France), emmanuelle.anceaume@irisa.fr 
occur, while the parallel time refers to as the successive number of steps each agent executes. Agents do not maintain nor use identifiers, however, for ease of presentation the agents are numbered $1,2, \ldots, n$. We denote by $C_{t}^{(i)}$ the state of agent $i$ at time $t$. The stochastic process $C=\left\{C_{t}, t \geq 0\right\}$, where $C_{t}=$ $\left(C_{t}^{(1)}, \ldots, C_{t}^{(n)}\right)$, represents the evolution of the population protocol. This means that, for every $i=1, \ldots, n$, we have $C_{0}^{(i)} \in\{0, m\}$. At each discrete instant $t$, two distinct indices $i$ and $j$ are uniformly chosen among $1, \ldots, n$, that is with probability $1 /(n(n-1))$. Once chosen, the couple $(i, j)$ interacts, and both agents update their respective local state $C_{t}^{(i)}$ and $C_{t}^{(j)}$ by applying the transition function $f$, leading to state $C_{t+1}$, given by $f\left(C_{t}^{(i)}, C_{t}^{(j)}\right)=\left(C_{t+1}^{(i)}, C_{t+1}^{(j)}\right)$ with

$$
\left(C_{t+1}^{(i)}, C_{t+1}^{(j)}\right)=\left(\left\lfloor\frac{C_{t}^{(i)}+C_{t}^{(j)}}{2}\right\rfloor,\left\lceil\frac{C_{t}^{(i)}+C_{t}^{(j)}}{2}\right\rceil\right) \text { and } C_{t+1}^{(r)}=C_{t}^{(r)} \text { for } r \neq i, j .
$$

We denote by $\ell$ the mean value of the sum of the entries of $C_{t}$ and by $L$ the row vector of $\mathbb{R}^{n}$ with all its entries equal to $\ell$, that is $\ell=\sum_{i=1}^{n} C_{t}^{(i)} / n$ and $L=(\ell, \ldots, \ell)$. We denote by $\|\cdot\|$ the Euclidean norm and by $\|\cdot\|_{\infty}$ the infinite one. Let $\lambda=\min \{\ell-\lfloor\ell\rfloor,\lceil\ell\rceil-\ell\}$, which is the distance between $\ell$ and its nearest integer. It is easily checked that we have $0 \leq \lambda \leq 1 / 2$. In Theorem 4 of [2], we dealt with the case where $\ell-\lfloor\ell\rfloor=1 / 2$. This case implies that $n$ is even. In the following theorem, we generalize these results to the case where $n$ is odd. We denote by $1_{\{A\}}$ the indicator function, which is equal to 1 if condition $A$ is true and 0 otherwise.

Theorem 2.1 For all $\delta \in(0,1)$, if $\lambda=\left(n-1_{\{n \text { odd }\}}\right) /(2 n)$ and if there exists a constant $K$ s.t. $\left\|C_{0}-L\right\| \leq K$ then, for every $t \geq(n-1)(2 \ln K-\ln \delta-\ln 2)$, we have

$$
\mathbb{P}\left\{\left\|C_{t}-L\right\|_{\infty}>\frac{n+1_{\{n \text { odd }\}}}{2 n}\right\}=\mathbb{P}\left\{\max _{1 \leq i \leq n} C_{t}^{(i)}-\min _{1 \leq i \leq n} C_{t}^{(i)}>1\right\} \leq \delta .
$$

The shadow process. We introduce what we call a shadow process of the stochastic process $C=\left\{C_{t}, t \geq 0\right\}$. This shadow process is a stochastic process denoted by $D=\left\{D_{t}, t \geq 0\right\}$ and defined at time $t=0$ by $D_{0}^{(i)}=C_{0}^{(i)}+1_{\left\{i \in B_{0}\right\}}$, where $B_{0}$ is a non empty subset of $b$ agents with $b \leq n-1$, i.e. $B_{0} \subset\{1, \ldots, n\}$ and $\left|B_{0}\right|=b$. For every $t \geq 1$, the shadow process $D_{t}$ is defined as process $C_{t}$, that is, when the couple $(i, j)$ is chosen to interact at time $t$, the vector $D_{t+1}$ is given by

$$
\left(D_{t+1}^{(i)}, D_{t+1}^{(j)}\right)=\left(\left\lfloor\frac{D_{t}^{(i)}+D_{t}^{(j)}}{2}\right\rfloor,\left\lceil\frac{D_{t}^{(i)}+D_{t}^{(j)}}{2}\right\rceil\right) \text { and } D_{t+1}^{(r)}=D_{t}^{(r)} \text { for } r \neq i, j .
$$


Both stochastic processes $C$ and $D$ behave identically and evolve following the same interactions. The only difference lies their initial values. Note that process $C$ is a part of the protocol but not process $D$ which is introduced only for the probabilistic analysis of $C$. As we did for process $C$, we denote by $\ell_{D}$ the mean value of the sum of the entries of $D_{t}$ and by $L_{D}$ the row vector of $\mathbb{R}^{n}$ with all its entries equal to $\ell_{D}$. Lemma 2.2 shows that if at time $t=0, D_{0}$ is in the shadow of $C_{0}$ then for any time $t \geq 0, D_{t}$ remains in the shadow of $C_{t}$.

Lemma 2.2 For all $t \geq 0$, there exists a non empty set $B_{t}$ of $b$ agents, i.e. $B_{t} \subset\{1, \ldots, n\}$ and $\left|B_{t}\right|=b$, such that for all $i \in\{1,2, \ldots, n\}$, we have

$$
D_{t}^{(i)}=C_{t}^{(i)}+1_{\left\{i \in B_{t}\right\}} .
$$

Lemma 2.3 For all $t \geq 0$, we have

$$
\left|\left\|D_{t}-L_{D}\right\|_{\infty}-\left\|C_{t}-L\right\|_{\infty}\right| \leq 1-\frac{1}{n} \text { and }\left|\left\|D_{t}-L_{D}\right\|-\left\|C_{t}-L\right\|\right|<\sqrt{n} \text {. }
$$

In Theorem 2.1, we obtained a first bound on the convergence time in the particular case where $\lambda=\left(n-1_{\{n \text { odd }\}}\right) /(2 n)$. This result together with Lemmas 2.2 and 2.3 are used to obtain general results, i.e. results which apply for any value of $\lambda$.

Theorem 2.4 For all $\delta \in(0,1)$, if there exists a constant $K$ such that $\left\|C_{0}-L\right\| \leq$ $K$, then, for every $t \geq(n-1)(2 \ln (K+\sqrt{n})-\ln \delta-\ln 2)$, we have

$$
\mathbb{P}\left\{\max _{1 \leq i \leq n} C_{t}^{(i)}-\min _{1 \leq i \leq n} C_{t}^{(i)}>2\right\} \leq \delta \text { and } \mathbb{P}\left\{\left\|C_{t}-L\right\|_{\infty} \geq \frac{3}{2}\right\} \leq \delta .
$$

\section{Application: Solving the Proportion Problem.}

We apply our results to the proportion problem.

Definition 3.1 (Proportion Problem.) A population protocol solves the proportion problem with precision $\varepsilon \in(0,1)$ and with probability at least $1-\delta$, $\delta \in(0,1)$, in $\tau(n, \varepsilon, \delta)$ interactions, if at any time $t \geq \tau(n, \varepsilon, \delta)$, any node is capable of computing $n_{A} / n$ with an $\varepsilon$-precision without the knowledge of the population size $n$.

We denote by $\gamma_{A}$ the proportion of nodes starting with $A$, i.e. $\gamma_{A}=n_{A} / n$, where $n_{A}$ is the number of nodes starting with $A$. The following theorem gives an evaluation of the first instant $t$ from which the distance between $C_{t}^{(i)} / m$ and $\gamma_{A}$ is less than a fixed $\varepsilon$ with any high probability $1-\delta$.

Theorem 3.2 For all $\delta \in(0,1)$ and $\varepsilon \in(0,1)$, by taking $m=\lceil 3 /(2 \varepsilon)\rceil$, we have, for all $t \geq(n-1)[\ln n-\ln \delta+2 \ln (2+1 / \varepsilon)+\ln (9 / 32)]$,

$$
\mathbb{P}\left\{\left|C_{t}^{(i)} / m-\gamma_{A}\right|<\varepsilon, \text { for all } i=1, \ldots, n\right\} \geq 1-\delta .
$$


Experimental results. We show how tight our bounds are by comparing the theoretical bound $\tau$ of the parallel convergence time to the results obtained via extensive simulations (see Figure 1). We introduce the parallel convergence time $\theta$ defined by $\theta=\inf \left\{t \geq 0\right.$ s.t. for all $\left.i,\left|C_{t}^{(i)} / m-\gamma_{A}\right|<\varepsilon\right\} / n$. We have run $N$ independent simulations of $\theta$ and stored the $N$ values of the parallel convergence times denoted by $\theta_{1}, \ldots, \theta_{N}$. The estimation of the first instant $t$ such that $\mathbb{P}\{\theta<t\} \geq 1-\delta$ is thus given by $\theta_{\lceil N(1-\delta)\rceil}$.

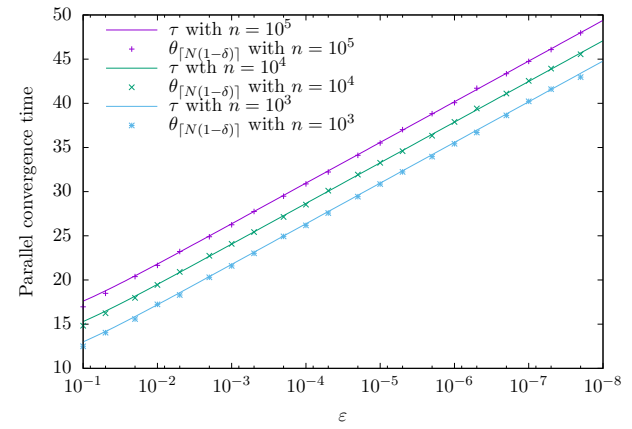

(a) $\theta_{\lceil N(1-\delta)\rceil}$ as a function of $\varepsilon$, with $\delta=$ $10^{-1}$ and $N=10^{4}$. From top to the bottom, we have $n=10^{5}, n=10^{4}$ and $n=10^{3}$ respectively.

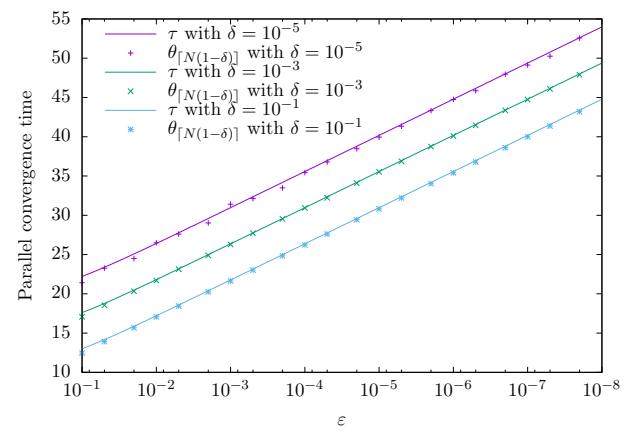

(b) $\theta_{\lceil N(1-\delta)\rceil}$ as a function of $\varepsilon$, with $n=10^{3}$ and $N=10^{6}$. From top to the bottom, we have $\delta=10^{-5}, 10^{-3}$ and $10^{-1}$ respectively.

Figure 1: Comparing the estimation $\theta_{\lceil N(1-\delta)\rceil}$ with the theoretical bound $\tau$ of the parallel convergence time (Theorem 3.2).

\section{References}

[1] D. Angluin, J. Aspnes, Z. Diamadi, M. J. Fischer, and R. Peralta, "Computation in networks of passively mobile finite-state sensors," Distributed Computing, vol. 18, no. 4, pp. 235-253, 2006.

[2] Y. Mocquard, E. Anceaume, and B. Sericola, "Optimal proportion computation with population protocols," in Proceedings of the IEEE NCA, 2016.

[3] Y. Mocquard, E. Anceaume, J. Aspnes, Y. Busnel, and B. Sericola, "Counting with population protocols," in Proceedings of the IEEE NCA, 2015.

[4] T. Sauerwald and H. Sun, "Tight bounds for randomized load balancing on arbitrary network topologies," in Proceedings of the IEEE FOCS, 2012.

[5] G. Cordasco and L. Gargano, "Space-optimal proportion consensus with population protocols," in Proceedings of the IEEE SSS, 2017. 